\title{
Operation of a single-GEM in noble gases at high pressures
}

\author{
F.D. Amaro ${ }^{a}$, A.S. Conceição ${ }^{a}$, J.F.C.A. Veloso ${ }^{b}$, L.C.C. Coelho ${ }^{\text {a }}$, L.M.P. Fernandes ${ }^{\mathrm{a}}$, \\ L.F. Requicha Ferreira ${ }^{a}$, J.A.M. Lopes ${ }^{\mathrm{a}, \mathrm{c}}$, J.M.F. dos Santos ${ }^{\mathrm{a}, *}$ \\ ${ }^{a}$ Physics Department, University of Coimbra, P-3004-516 Coimbra, Portugal \\ ${ }^{\mathrm{b}}$ Physics Department, University of Aveiro, P-3810-193 Aveiro, Portugal \\ ${ }^{\mathrm{c}}$ Instituto Superior de Engenharia de Coimbra, Apartado 4065, P-3030-199 Coimbra, Portugal
}

Available online 4 April 2007

\begin{abstract}
We report the performance of a single-Gas Electron Multiplier (GEM) operating in pure Ar, Xe, and in Ar-501mbar Xe mixtures, in the range of 1-7lbar. The maximum gain and voltage that can be applied to the GEM are investigated as a function of filling pressure and compared to the results obtained with triple-GEM and MHSP (Micro Hole and Strip Plate) multipliers. The maximum gain achieved at $1 \mathrm{lbar} \mathrm{Xe}$ is about 103, presenting a fast decrease with pressure to values around 300, 50 and 10 at 2, 3 and 5lbar, respectively. Gains around 100 were achieved in Ar up to 4lbar, decreasing to values of few tens at 6lbar. On the other hand, gains around 500 can be achieved in Ar-501mbar Xe mixtures up to 5lbar, presenting a fast reduction at higher pressures due to the limitations on the maximum gain imposed by the GEM discharge limit. Nevertheless, gains above 100 can be obtained for pressures between 6 and $7 \mathrm{lbar}$, indicating a good potential for neutron detection.
\end{abstract}

(C) 2007 Elsevier B.V. All rights reserved.

PACS: $29.40 .-\mathrm{n} ; 29.40 . \mathrm{Cs} ; 85.60 . \mathrm{Gz}$

Keywords: Gas avalanche multipliers; GEM; MHSP; Triple GEM; High pressure; Noble gas

\section{Introduction}

The operations of triple-Gas Electron Multiplier (GEM) and Micro Hole and Strip Plate (MHSP) electron multipliers in high-pressure noble gases have been investigated in detail through the last 6 years [1-6]. Applications to cryogenic double-phase detectors, for neutrino physics and dark matter search [7,8], and to neutron detection [9] have been envisaged, but they also present good alternatives for hard X-ray detection applications such as digital radiography, synchrotron radiation studies, crystallography and astrophysics.

Noble gases have the important advantages of simple handling and purification procedures, and the ability of not presenting ageing under charge avalanche. This allows the design of sealed detectors with stable long-term operation.

\footnotetext{
*Corresponding author. Tel.: + 351239410667 ; fax: + 351239838850 .

E-mail addresses: fcjam@gian.fis.uc.pt (J.A.M. Lopes), jmf@gian.fis.uc.pt (J.M.F. dos Santos).
}

However, charge multiplication in noble gases is strongly limited by photon-mediated secondary effects, and organic quenchers have been added to pure noble gases to suppress the UV-scintillation occurring in the electron avalanches. Recently, the feasibility of high-gain operation of multiGEM cascades in pure noble gases was demonstrated. The avalanche confinement within the microstructure holes hinders photon-mediated secondary processes, allowing high gains to be achieved even in highly UV-scintillating gases $[10,11]$.

However, studies have revealed that the maximum gain that could be achieved in triple-GEM and MHSP electron multipliers drops with increasing gas pressure, for heavy noble gases, $\mathrm{Xe}, \mathrm{Kr}$ and $\mathrm{Ar}$. This is due to the less efficient charge transfer between amplification stages and to the reduction of the electron-impact ionisation yield. The reduction in the ionisation efficiency is due to the decrease of the reduced electric field in the avalanche region, limited by the total voltage that can be applied to the multipliers before discharge, as the pressure increases, cf. Ref. [6] and 
references therein. It was demonstrated that MHSPs yield higher gains for $\mathrm{Xe}, \mathrm{Kr}$ and $\mathrm{Ar}$ at high pressures than triple-GEMs [6], presenting a much slower rate for gain reduction with increasing pressure. For dense light noble gases, $\mathrm{Ne}$ and $\mathrm{He}$, other mechanisms such as associative ionisation and/or Penning ionisation with impurities predominate over electron-impact ionisation and tripleGEM achieves rather high gains, of the order of $10^{5}$, which are almost pressure independent $[12,13]$.

Single-GEM operation at high pressure was only studied in detail for $\operatorname{Kr}$ [3] and presents an even slower rate for gain reduction with increasing pressure than MHSPs [6]. Compared to triple-GEM, single-GEM presents higher gains for $\mathrm{Kr}$ pressures above 4 bar [3]. On the other hand, the gain achieved with the single-GEM in $\mathrm{Kr}$ operated at atmospheric pressure, $\sim 500$, is more than two orders of magnitude lower than that achieved with the MHSP, reducing this difference to about one order of magnitude at pressures of 6 bar, with charge gains around 200. However, single-GEM is a good alternative for high-pressure operation in applications where high gain is not a requirement, e.g. for neutron detection, a gain of $\sim 100$ is sufficient, and where the decoupling of the readout system from the amplification stage is an advantage for detector operation, in opposition to MHSPs.

In this work, we investigate in detail the performance of a single-GEM operating in high-pressure $\mathrm{Xe}, \mathrm{Ar}$ and in Ar-50 mbar Xe mixtures (with interest for neutron detection [9]). The gain and maximum voltage that can be applied to the GEM are determined as a function of filling pressure, in the range of 1-7 bar, and compared to the results obtained with triple-GEM and MHSP multipliers.

\section{Experimental set-up}

The GEMs used in this work were manufactured at CERN and have standard dimensions: $50-\mu \mathrm{m}$ Kapton with $5-\mu \mathrm{m}$ copper clad on both sides and with bi-conical holes of 50 and $70 \mu \mathrm{m}$ in the Kapton and copper, respectively, arranged in a hexagonal layout of $140-\mu \mathrm{m}$ edges. The GEMs active area is $2.8 \times 2.8 \mathrm{~cm}^{2}$. A stainless-steel detector body was built to accommodate the GEM; the absorption/ drift region and the induction region gaps are 4- and 3-mm wide, respectively, Fig. 1. Macor pieces, simply glued with low vapour-pressure epoxy (Tra-Con 2116) to the stainlesssteel body, were used for insulating the feedthroughs of the detector biasing. The GEM was mounted on a Macor frame to keep it stretched and to provide the electrical contacts to the GEM surfaces. The detector window was made of aluminised Mylar foil (25- $\mu \mathrm{m}$ thick) glued to the detector body with the same epoxy.

The detector was vacuum pumped down to pressures of $10^{-5} \mathrm{mbar}$ and, afterwards, filled with noble gases at different pressures without backing; it was sealed off during the measurements. The gas purity was maintained using non-evaporable getters (SAES St707), heated up to

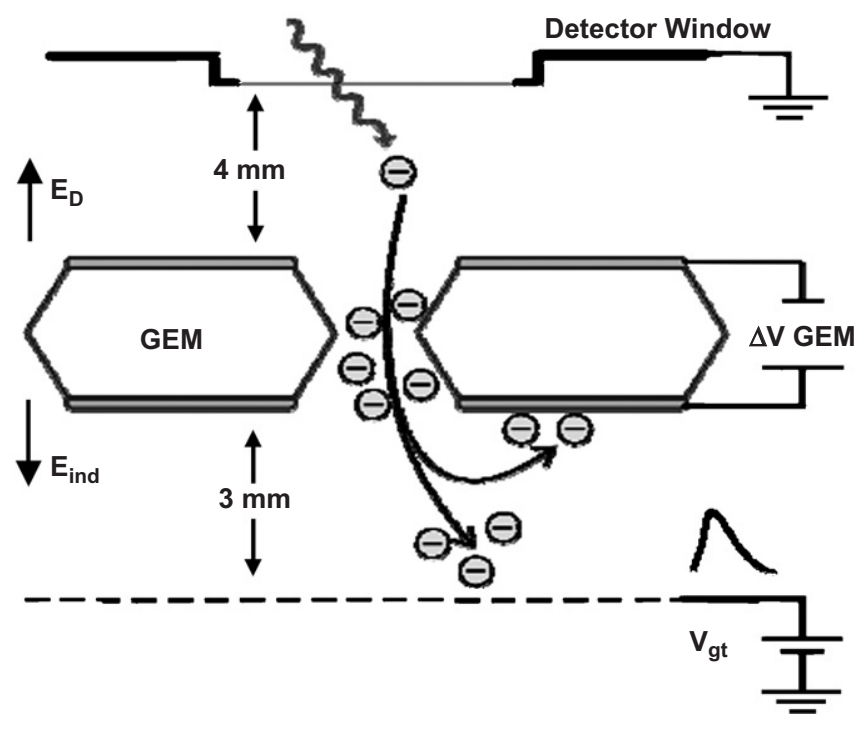

Fig. 1. Schematic diagram of the single-GEM detector used in this work.

about $150{ }^{\circ} \mathrm{C}$ and placed in a small volume connected to the detector one.

The radiation window and the detector body are grounded, while the different electrodes, GEM top and bottom electrodes and the induction mesh, are polarised independently. The voltage in the GEM top electrode determines the drift field; the voltage difference between the GEM top and bottom electrodes, $\Delta V_{\mathrm{GEM}}$, determines the avalanche gain in the holes; and the voltage difference between the GEM-bottom electrode and the induction mesh determines the induction field.

The detector was irradiated with $22.1 \mathrm{keV} \mathrm{X-rays} \mathrm{from} \mathrm{a}$ ${ }^{109} \mathrm{Cd}$ source, allowing a clear separation of the peak distribution from the electronic noise tails for reduced detector gains. The primary electron clouds resulting from $\mathrm{X}$-ray interactions in the drift region are focused into the holes where they undergo avalanche multiplication, Fig. 1; the avalanche electrons are extracted out of the holes and are collected in both the GEM-bottom surface and induction mesh. The signals from the mesh were fed through a Canberra 2006 preamplifier (sensitivity of $\left.1.5 \mathrm{~V} \mathrm{pC}^{-1}\right)$ and a Tennelec TC243 amplifier ( $4 \mu$ s shaping time) to a Nucleus PCA2 1024 multichannel analyser. The electronic chain sensitivity was calibrated for absolute gain determination, using a calibrated capacitor directly connected to the preamplifier input as well as to a precision pulse generator. The gains were determined from the peakposition of the pulse-height distributions.

\section{Experimental results and discussion}

Throughout the measurements, the reduced drift field, determined by the GEM upper electrode voltage, was kept at values between 0.2 and $0.3 \mathrm{kV} \mathrm{cm}^{-1} \mathrm{bar}^{-1}$ for pure $\mathrm{Ar}$ and Ar-50 mbar Xe mixtures, and $\sim 0.5 \mathrm{kV} \mathrm{cm}^{-1} \mathrm{bar}^{-1}$ for pure Xe. 
As referred to above, the avalanche electrons are collected in the GEM-bottom electrode and in the induction electrode. Therefore, the GEM total gain should include the charge collected in both the electrodes. However, in practical cases, the effective gain of the GEM is related with the charge collected in the induction plane and depends on the induction electric field. At atmospheric pressure, induction fields as high as $3 \mathrm{kV} \mathrm{cm}^{-1}$ result in electron collection efficiencies around 50\% [14], i.e. in an effective gain that is half of the total gain achieved in the electron avalanches in the holes. In addition, the induction electric field has a small effect on the GEM total gain, which increases about $20-30 \%$ as the induction electric field increases from 0 to $8 \mathrm{kV} \mathrm{cm}^{-1}$ for Ar-based mixtures [15]. In our work, the maximum voltage that could be supplied to the induction mesh was limited by sparking between mesh and detector body, imposing an upper limit to the value of the induction field that could be operated.

In Fig. 2, we present the GEM total gain (solid symbols) and effective gain (open symbols) as a function of voltage difference across the holes, $\Delta V_{\mathrm{GEM}}$, for pure Ar (Fig. 2a) and Xe (Fig. 2b), at filling pressures of 1-6 bar. In each set of measurements, $\Delta V_{\mathrm{GEM}}$ was gradually increased up to the onset of microdischarges. The GEM total gain was

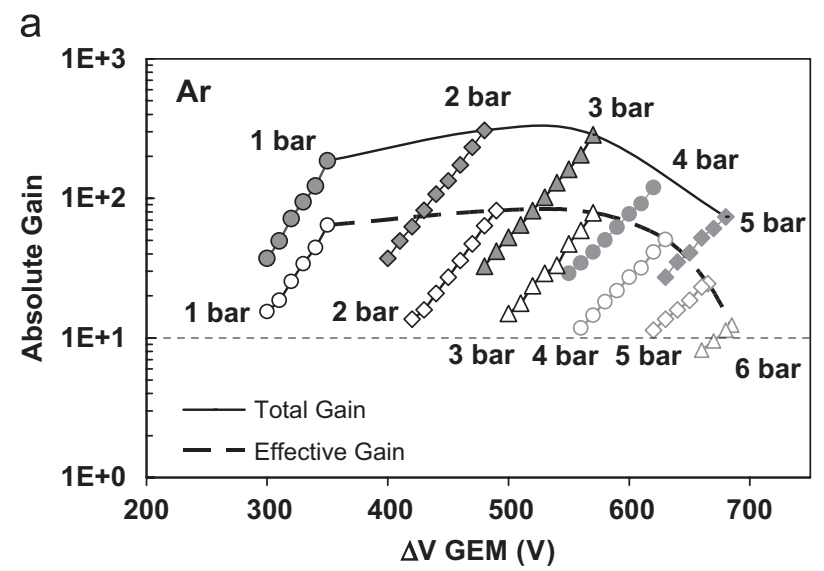

b

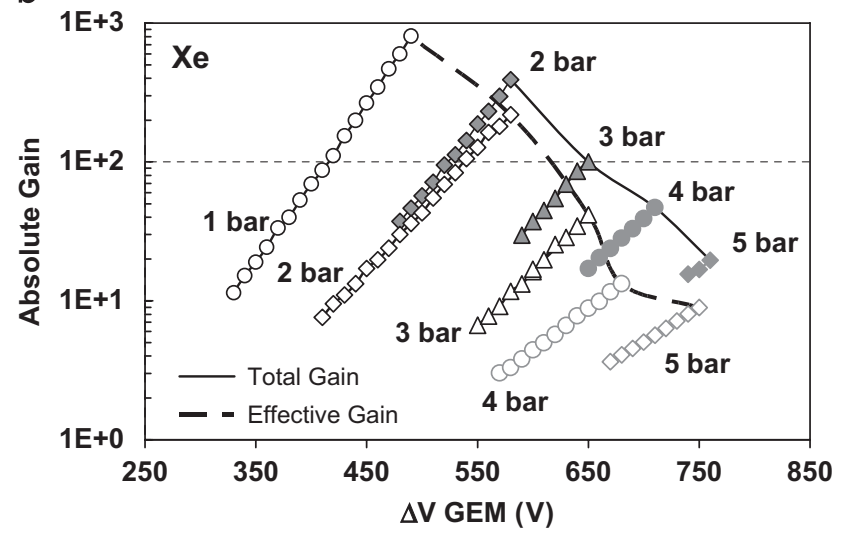

Fig. 2. GEM total and effective gain as a function of voltage difference applied across the GEM, $\Delta V_{\mathrm{GEM}}$, for argon (a) and xenon (b) and for different filling pressures. measured by interconnecting the GEM-bottom electrode and the induction electrode. The curves exhibit the characteristic exponential avalanche growth. As in MHSPs, Fig. 2 shows different trends of the gain dependence on pressure for Ar and Xe: while for Ar, the maximum achievable gain presents a small dependence on the pressure up to 4 bar, decreasing by a factor of two as the pressure increases to 5 and, then, to 6 bar; for xenon, the maximum gain presents a fast decrease from 1 to 3 bar and a slower decrease for higher pressures, presenting an overall drop of two orders of magnitude at 5 bar. The maximum effective gains we could achieve in $\mathrm{Ar}$ were around 100 . Gains of the order of $10^{3}$ were only achieved for 1 bar Xe; the fast reduction in gain of a factor of 4 at 2 bar and, again, at 3 bar may compromise the application of a Xe single-GEM counter to hard X-ray detection.

The data in Fig. 2 show two- to four-fold lower effective gains when compared to the total gains, as a consequence of the low reduced electric fields used in the induction region: for $\mathrm{Ar}, 2 \mathrm{kV} \mathrm{cm}^{-1}$ at $1 \mathrm{bar}$, decreasing to 0.8 and $0.4 \mathrm{kV} \mathrm{cm}^{-1} \mathrm{bar}^{-1}$ at 2 and 6 bar, respectively, and for $\mathrm{Xe}, 4 \mathrm{kV} \mathrm{cm}^{-1} \mathrm{bar}^{-1}$ for 1- and 2-bar fillings and $1 \mathrm{kV} \mathrm{cm}^{-1} \mathrm{bar}^{-1}$ for the other pressures. Therefore, in practical applications, the effective gains that can be achieved with single-GEM operating in pure Ar and Xe do not differ much from those depicted in Fig. 2.

As discussed in [9], noble gas Ar-based mixtures with a small Xe content may be an alternative as a stopping gas for neutron detection. These mixtures are known to be Penning mixtures, providing high gains at relatively low voltages [1,5], which is of particular interest for high pressure operation. In addition, they will be an advantage for sealed detectors with only noble gas filling, simple to purify and handling and not subject to ageing. A mixture of Ar-50 mbar Xe presents $\gamma$-sensitivities as low as for $\mathrm{CF}_{4-}$ based mixtures [9] and MHSP operating in such atmosphere could operate with gains above $10^{3}$ under pressures up to 7 bar, without significant reduction in the maximum achievable gain [5]. Therefore, single-GEM operation in such atmospheres can be an interesting alternative, in case it can deliver gains above 100 at 6 bar.

We investigate the dependence of the GEM effective gain as a function of voltage difference across the holes, $\Delta V_{\mathrm{GEM}}$, for Ar-50 mbar Xe mixtures for filling pressures of 1-7.4 bar, for the same biasing conditions as those set for Ar (Fig. 3). Like for the MHSP, the GEM effective gain does not depend significantly on the pressure until the maximum voltage the GEM can support in this mixture is reached, presenting an abrupt drop for higher pressures. Gains around 300 are obtained for filling pressures up to 5 bar, dropping to 120 and 40 as the pressure increases to 6 and 7.4 bar, respectively. Taking into account that increasing the induction field results in a gain increase that can easily be a factor of two higher than those depicted in Fig. 3 , it becomes clear that the GEM can reach gains above 100 in Ar-50 mbar Xe mixtures for filling pressures between 6 and 7 bar, demonstrating the feasibility of applying a 


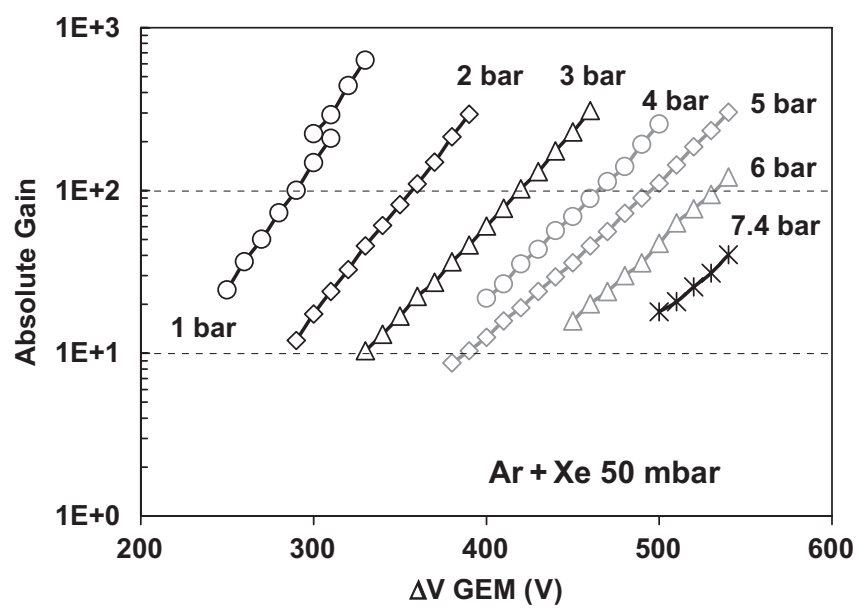

Fig. 3. GEM effective gain as a function of voltage difference applied across the GEM, for argon-50 mbar xenon and for filling pressures ranging from 1 to $7.4 \mathrm{bar}$.

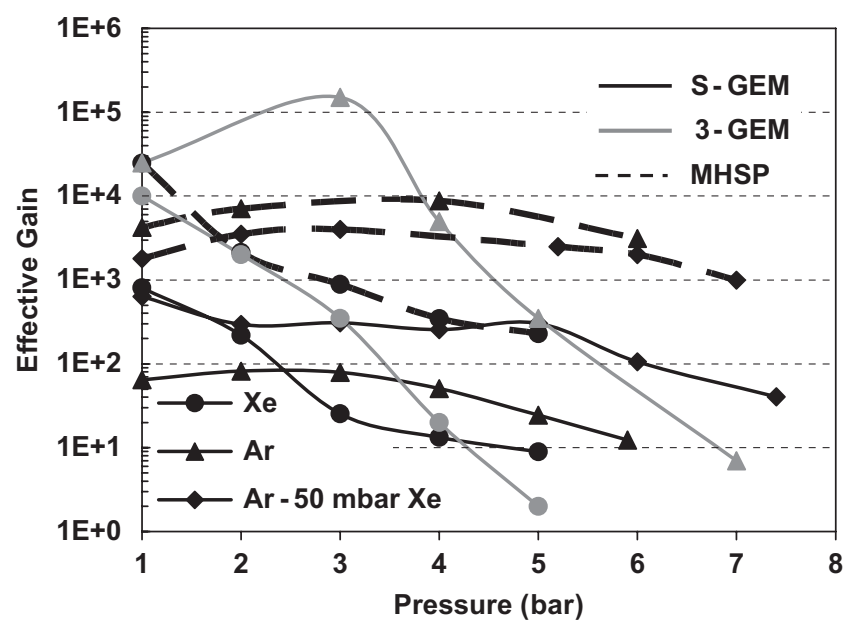

Fig. 4. Maximum gain of different detectors as a function of gas pressure for the different noble gases fillings: solid dark lines-single-GEM detector [this work]; grey solid lines-triple-GEM detector [3] and broken lines-MHSP detector [6].

single-GEM to neutron detection, using this gas mixture as a stopping gas.

Fig. 4 summarises the maximum gain achieved with a single-GEM as a function of gas pressure for $\mathrm{Ar}$, Xe and Ar-50 mbar Xe mixture. For comparison, we include the maximum gains achieved with a triple-GEM [12] and an MHSP [6]. Compared with the triple-GEM multiplier, the single-GEM presents much lower gains but, also, a much slower decrease of the maximum gain with increasing pressure, leading to similar gains for pressures around 4 and 7 bar for Xe and Ar, respectively. Nevertheless, at these pressures and above, the gains are already too small, around or below 10, to be of practical use. On the other hand, the MHSP presents much higher gains and similar gain dependence with pressure when compared to a singleGEM.

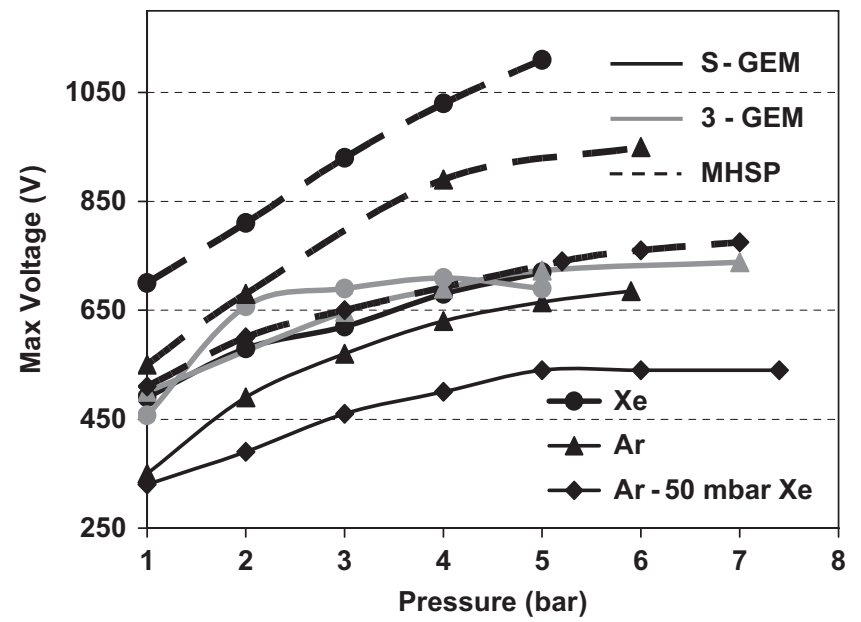

Fig. 5. Maximum operation voltage as a function of pressure for the different noble gas fillings: solid dark lines - single-GEM detector [this work]; grey solid lines - triple-GEM detector [3] and broken lines-MHSP detector [6].

The main reason for the above differences is related with the voltage that can be applied to the GEMs and MHSP multipliers as the pressure increases. Fig. 5 presents the maximum operation voltage that can be applied across each GEM in a single- and triple-GEM multiplier and to an MHSP in Ar, Xe and Ar-50 mbar Xe mixture. When the pressure increases, the voltage saturates faster in a tripleGEM cascade than in a single-GEM and MHSP multipliers. This effect is attributed to the onset of feedback effects due to ion-induced electron emission. The noble gas ions produced in the electron avalanches are neutralised in the copper electrodes of the microstructure surfaces, inducing secondary electron emission. This limits the maximum applicable voltage in triple-GEM [12], being the effect considerably reduced in single-element multipliers. On the other hand, the total voltage applied to the MHSP is much higher, when compared to that applied to a single-GEM.

In all cases, the reduced electric field, $E / p$, in the avalanche region, limited by the total voltage that can be applied to the multipliers before discharge, decreases as the pressure increases. Since electron avalanches in Ar and Xe are determined by the electron-impact mechanism, the maximum gain drops for high pressures, since the maximum applied voltage does not increase as fast as pressure, resulting in a reduction in the Towsend coefficient [14]. This is even more noticeable for the single-GEM operation in Ar-50 mbar Xe mixture, where the maximum applied voltage completely saturates for pressures above 5 bar and the avalanche gain presents a very fast decrease as the pressure increases.

\section{Conclusions}

We have investigated the characteristics of a single-GEM operated in $\mathrm{Ar}, \mathrm{Xe}$ and Ar-50 mbar Xe mixtures at pressures ranging from 1 to 7 bar. The maximum gains 
achieved in Ar prior to the onset of microdischarges are low, $\sim 100$, presenting a small variation with pressure up to 4 bar and decreasing to values of few tens at 6 bar. On the other hand, the maximum gains obtained in pure xenon are about $10^{3}$ at 1 bar and present a fast decrease with pressure to values around 300 and 50 at 2 and 3 bar, respectively, being $\sim 10$ at 5 bar. Compared with triple-GEM, the single-GEM presents much lower gains and a slower decrease of the maximum gain with increasing pressure, achieving gains similar to triple-GEM only for pressures around 4 and $7 \mathrm{bar}$, for $\mathrm{Xe}$ and $\mathrm{Ar}$, respectively. Nevertheless, for these pressures, the gains are already too small, around or below 10, to be of practical use.

On the other hand, the operation of single-GEM in high pressure Ar-50 mbar Xe mixture is more interesting, with potential application to neutron detection. Gains around 500 can be obtained up to 5 bar. Above 5 bar limitations on the maximum gain are imposed by the GEM discharge limit. Nevertheless, gains above 100 can be obtained for pressures between 6 and 7 bar.

The single-GEM presents trends similar to the MHSP for the variation of the maximum gain as a function of pressure, but the gains achieved with single-GEM are more than one order of magnitude lower than those obtained with the MHSP.

\section{Acknowledgments}

This work was supported by Project POCTI/FNU/ 50360/2002 through FEDER and FCT (Lisbon) programs.

\section{References}

[1] A. Buzulutskov, A. Breskin, R. Chechik, G. Garty, F. Sauli, L. Shekhtman, Nucl. Instr. and Meth. A 443 (2000) 164 and references therein.

[2] A. Bondar, A. Buzulutskov, L. Shekhtman, Nucl. Instr. and Meth. A 481 (2002) 200.

[3] V. Aulchenko, A. Bondar, A. Buzulutskov, L. Shekhtman, R. Snopkov, Yu. Tikhonov, Nucl. Instr. and Meth. A 513 (2003) 256 and references therein.

[4] A. Orthen, H. Wagner, H.J. Besch, S. Martoiu, R.H. Menk, A.H. Walenta, U. Werthenbach, Nucl. Instr. and Meth. A 512 (2003) 476.

[5] F.D. Amaro, J.F.C.A. Veloso, J.M. Maia, A. Breskin, R. Chechik, J.M.F. dos Santos, Nucl. Instr. and Meth. A 535 (2004) 341.

[6] F.D. Amaro, J.F.C.A. Veloso, A. Breskin, R. Chechik, J.M.F. dos Santos, J. Inst. 1 (2006) P04003.

[7] A. Bondar, A. Buzulutskov, D. Pavlyuchenko, R. Snopkov, Y. Tikhonov, Nucl. Instr. and Meth. A 548 (2005) 439.

[8] A. Bondar, A. Buzulutskov, A. Grebenuk, D. Pavlyuchenko, R. Snopkov, Y. Tikhonov, Nucl. Instr. and Meth. A 556 (2006) 273.

[9] J.F.C.A. Veloso, F.D. Amaro, J.M.F. dos Santos, J.A. Mir, G.E. Derbyshire, R. Stephenson, N.J. Rhodes, E.M. Schooneveld, IEEE Trans. Nucl. Sci. NS-51 (2004) 2104.

[10] A. Buzulutskov, L. Shekhtman, A. Bressan, A. Di Mauro, L. Ropelewski, F. Sauli, S. Biaggi, Nucl. Instr. and Meth. A 433 (1999) 471

[11] A. Breskin, A. Buzulutskov, R. Chechik, Nucl. Instr. and Meth. A 483 (2002) 670.

[12] A. Buzulutskov, Nucl. Instr. and Meth. A 494 (2002) 148 and references therein.

[13] A. Buzulutskov, J. Dodd, R. Galea, et al., Nucl. Instr. and Meth. A 548 (2005) 487.

[14] R. Chechik, A. Breskin, G.P. Guedes, D. Mörmann, J.M. Maia, V. Dangendorf, D. Vartsky, J.M.F. dos Santos, J.F.C.A. Veloso, IEEE Trans. Nucl. Sci. NS-51 (2004) 2097.

[15] J.M. Maia, "Study and development of a novel micropattern aseous radiation detector: The Microhole and strip plate," Ph.D. Thesis, University of Coimbra (2005); to be submitted to J Instrum (2007). 